\title{
Applying the SVM method in an engineering optimization problem
}

\author{
Attila Vámosi \\ Faculty of Engineering, University of Debrecen \\ vamosi.attila@eng.unideb.hu
}

\begin{abstract}
This work aims to demonstrate that machine learning is suitable for solving engineering problems. In this work Support Vector Machine (SVM) method has been chosen to investigate a specific engineering optimization problem.

Engineering rubber parts must have a predefined load-displacement characteristics under load. This characteristics depends on three parameters of the geometric shape. The aim is to find the rubber part geometry resulting the minimum difference between the desired and the initial load-displacement characteristics. This area can be named as the difference of work $(\Delta \mathrm{W})$. The objective is to minimize this area, therefore we have to find a regression function for delta work.

SVM can be used for classification and regression as well. The linearly non-separable cases can be solved by using the "kernel trick", and using an $\varepsilon$-insensitive loss function the regression task can be solved, too. The application of the method has 4 steps. First, we determine the training points and generate the set of training data. Then we choose the Kernel function and determine hyper-parameters. We search the optimal hyper-parameters using grid search and n-fold cross-validation to avoid overfitting. Finally, we executing the regression with the optimal hyper-parameters, and we get the resulted regression function.

In this example 27 training points have been selected and we used Radial Basis Function kernel. After the grid search we get the optimal value of the three hyper-parameters. With the resulted regression function can we calculate the minimum of the work difference and finally we can determine the optimal geometric parameters.
\end{abstract}

Keywords: machine learning, optimization, regression, Support Vector Machine, SVM 


\section{References}

[1] Mankovits, T. - Kocsis, I. - Portik, T. - Szabó, T. - Páczelt, I.: Shape Design of Rubber Part Using FEM, International Review of Applied Sciences and Engineering, 4(2), 2012., pp. 85-94.

[2] Mankovits, T. - Szabó, T.: Finite Element Analysis of Rubber Bumper Used in Airsprings. Procedia Engineering, 48, 2012, pp. 388-395.

[3] Mankovits, T. - Szabó, T. - Kocsis, I. - Páczelt, I.: Optimization of the Shape of AxiSymmetric Rubber Bumpers, Strojniski vestnik - Journal of Mechanical Engineering, 60(1), 2014, pp. 61-71. 